\title{
Research for health equity policy - being strategic about reality
}

\author{
Jinhee Kim, Evelyne de Leeuw, Patrick Harris
}

\section{The issue}

The importance of the social determinants of health and health equity is well established. Interventions targeting upstream policies at the systems level are known to be effective over downstream interventions targeted at the individual level. However, the disjunction prevails between research on the effectiveness of upstream approaches and descriptive research targeting downstream interventions - there is a 'lifestyle drift' in policy research. ${ }^{1,2}$

\section{What we already knew}

Although the association between the systemic factors and health equity is commonly recognised, good reviews of evidence at those more distal levels of influence are still rare. ${ }^{3,4}$ One explanation for this lack of good reviews is that policymaking is a non-linear, dynamic process that is difficult and expensive to capture in terms of long term outcomes. ${ }^{1,2}$

\section{New evidence suggests...}

A health political science approach to investigating the disjunction between research and policy provides valuable insights. Instead of 'factual evidence', Smith (2014) found that 'research-

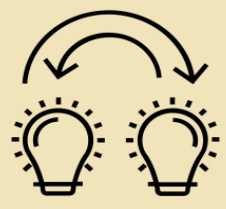

informed ideas' travel between research and policy.

In this process, research actors and policy participants emphasise those aspects of the evidence that complement prevailing (perceived) realities.

- $\quad$ Research-informed ideas on health inequities remain aligned with the biomedical model of health. The more challenging aspects that drive a social model of health are downplayed or omitted.
- Researchers frame their evidence to maintain credibility with funders and users of their work. Radical innovations do not fit with the relevant incentive mechanisms.

- The architecture of institutions that generate policies tend to reflect a reductionist biomedical and clinical thinking. These approaches fail to effectively address complex systemic conditions that create equity.

- Additionally, there is a perception that egalitarian policy is socially and electorally less attractive.

- Therefore, policies and research that feed into the existing institutional architecture naturally align with dominant thought and power.

\section{The strength of the evidence in practice}

Smith (2014) interviewed 112 key actors including researchers, civil servants, policy advisors, journalists, politicians and research funders to investigate the interplay of ideas between research and policy in the UK. ${ }^{1}$ The findings are supported by Baker et al. (2018) review of 48 peer-reviewed publications to analyse factors that increase or decrease the 'probability' of health equity reaching a government agenda. ${ }^{2}$

\section{References}

Smith, K. (2014). The politics of ideas: the complex interplay of health inequalities research and policy." Science and Public Policy 41,(5): 561-74

Baker, P. et al. (2018) What enables and constrains the inclusion of the social determinants of health inequities in government policy agendas? A narrative review. " Int J Health Policy Manag 7,(2): 101-11.

Bambra, C. et al. (2010). Tackling the wider social determinants of health and health inequalities: evidence from systematic reviews. J Epidemiol Community Health, 64, 284-291.

Naik, Y. et al. (2019). Going upstream - an umbrella review of the macroeconomic determinants of health and health inequalities. BMC Public Health، 19: 1678.

\section{Putting it into policy and action}

Researchers, practitioners and policymakers can apply a critical view in processing information on health equity issues. They ought to be aware that they are ideas which have been framed to fit the values and interests of neoliberal and biomedical actors and institutions that promote policies for the benefit of a select few rather than the population at large.

To promote the upstream policies and seek policy change, it may be effective to identify other research and policy actors who share those similar ideas on health equity and build strong epistemic networks to provide a counter narrative to the dominant discourse. Small 'wins' for equity may need to go hand-in-hand with strategically opportunistic other pursuits. 